\title{
Sometimes, the Least Demanding Solution Is the Most Suitable: A Careful Survey of NMR Spectra of a Phosphonium Salt Accounted for Its "Unusual Wittig Reaction"
}

\author{
Anna Fantinati, ${ }^{\dagger}$ Paolo Marchetti, ${ }^{\dagger}$ Claudio Trapella, ${ }^{* \bullet}$ and Vinicio Zanirato \\ Department of Chemical and Pharmaceutical Sciences, University of Ferrara, Via Fossato di Mortara 17, I-44121 Ferrara, Italy
}

Supporting Information

ABSTRACT: Careful product analysis in combination with appropriate supporting experiments unambiguously proves that in contrast to what was previously reported, generation of the phosphonium salt from a $\beta$ cyclocitral-derived allylic alcohol and $\mathrm{PPh}_{3} \mathrm{HBr}$ takes place via a protondriven elimination/addition path.

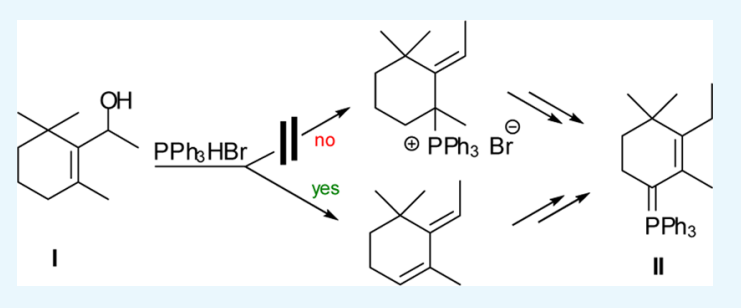

\section{INTRODUCTION}

In 2008, (E)-4-((3-ethyl-2,4,4-trimethylcyclohex-2-enylidene)methyl)benzoic acid 1 was unexpectedly obtained by Das and Kabalka ${ }^{1}$ during their preparation of novel analogues of alltrans-retinoic acid by changing the flexible alkene backbone into a more rigid phenyl ring. This scaffold has been later widely utilized by the same group both in the field of retinoids $^{2,3}$ and in that of mitochondria-associated, granulocyte-macrophage colony-stimulating factor signaling inhibitors, wherein guanidinamide $2^{4 a}$ represents the lead of a small library of molecules (Figure 1).

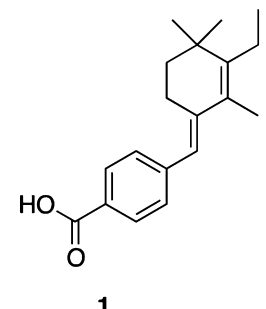

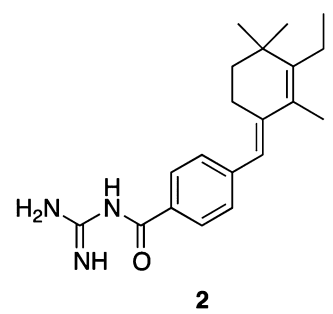

2
Figure 1. Structure of the acid precursor and the lead compound as MAGMAS inhibitors.

The relevant biological profile of compound 2, coupled with its ready availability, led us to use this scaffold for the construction of a small library of structural analogues in the context of an ongoing chemical biology project. ${ }^{4 \mathrm{~b}}$ Accordingly, the precursor ester $\mathbf{6}$ of acid $\mathbf{1}$ has been conveniently synthesized starting from $\beta$-cyclocitral 3 along the pathway outlined in Scheme 1.

\section{RESULTS AND DISCUSSION}

In detail, the reaction of 4-formylbenzoic acid methyl ester with phosphorus ylides featuring the $\beta$-cyclocitral skeleton afforded compounds 6 in place of the planned regioisomeric dienes 5. Thus, different from their expectations, the phosphonium salts 9, not 7, were the precursors of the pivotal ylides for the Wittig olefination reaction.

The reaction of allylic alcohols with triphenylphosphine hydrobromide $\left(\mathrm{Ph}_{3} \mathrm{P} \cdot \mathrm{HBr}\right)$ is a well-established method for the direct preparation of allyl triphenylphosphonium bromide salts. Notably, in the case of tertiary allyl alcohols acting as the substrates, the phosphonium salts, where the least hindered electrophilic carbons intercept the phosphine nucleophile, are usually observed as the major products. ${ }^{5}$ In the specific case of allylic alcohols 4, the formation of the two regioisomeric phosphonium salts 7 and $\mathbf{1 1}$ was anticipated. The former phosphonium salt resulted from the ipso substitution of bromide, whereas the latter phosphonium salt resulted from its allylic displacement (Scheme 2). Thus, 7 would have given the dienes 5, whereas 11, lacking in $\alpha$-proton, would not have been exploitable in Wittig olefination. Thus, the authors are faced with an "unusual Wittig reaction".

In the primary ${ }^{6}$ as well as in successive papers, ${ }^{7}$ the authors speculated that under basic conditions $(n-\mathrm{BuLi}$ or $t$-BuONa), the salt $\mathbf{1 1}$ could originate the pivotal ylide $\mathbf{1 0}$ for the "Wittig olefination" (Scheme 2). They envisioned that deprotonation at methylene beta carbon of 11 triggered a 1,2-phosphorus migration followed by an intermolecular proton shift giving ylides 10. The subsequent in situ reaction with the aromatic aldehyde led to the unexpected dienes 6 . In support of the postulated mechanism, they reported the NMR spectral characterization of the "unusual Wittig salt" $11(\mathrm{R}=\mathrm{Me})$ that could be isolated as a yellow solid after recrystallization from methanol/ether (1:6).

In the context of an ongoing chemical biology project, we selected compound $6(\mathrm{R}=\mathrm{Me})$ as a scaffold from which to prepare a small library of guanidine derivatives. With the aim of

Received: June 4, 2018

Accepted: July 6, 2018

Published: July 19, 2018 


\section{Scheme 1. Wittig Olefination}<smiles>CC1=C(C=O)C(C)(C)CCC1</smiles>

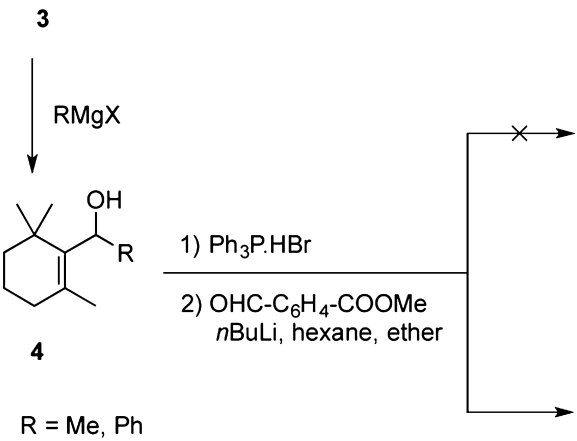<smiles>[R]C(=Cc1ccc(C(C)=O)cc1)C1=C(C)CCCC1(C)C</smiles><smiles>CCCCCCCCC(=Cc1ccc(C(C)=O)cc1)CC</smiles><smiles>[R]C(=[PH2]c1ccc([18OH])cc1)C1=C(C)CCCC1(C)C</smiles><smiles>[R]CC1=C(C)C([Pb])([Pb])CCC1(C)C</smiles>

7

8

9

10

Scheme 2. Mechanism of the Unusual Wittig Reaction

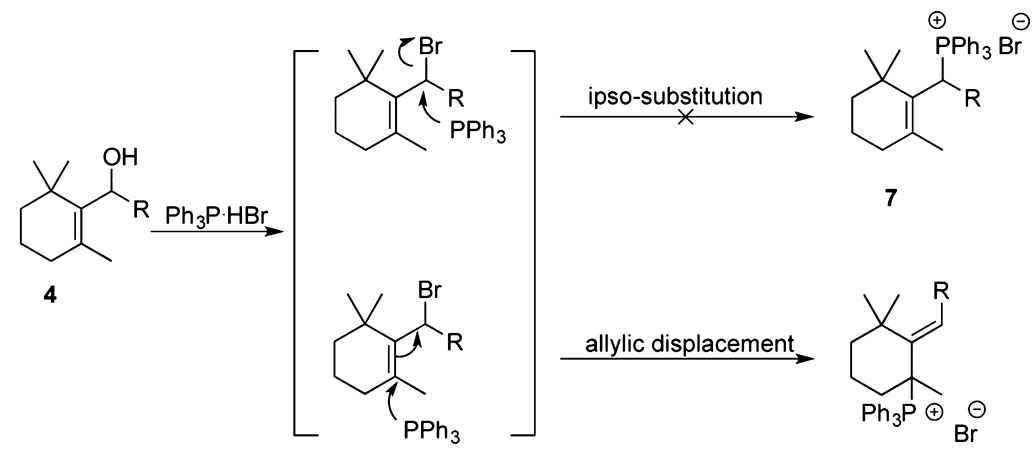

11

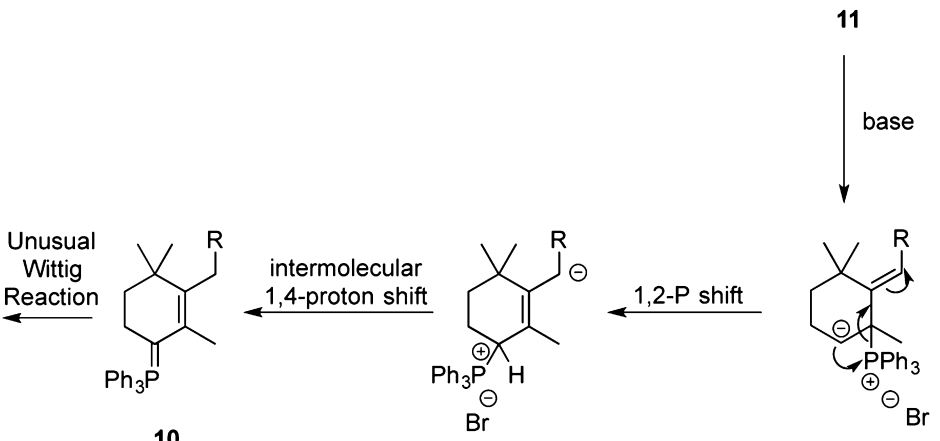

arriving to the 1,3-diene system incorporating the $\beta$-cyclocitral skeleton, we found it convenient to follow the Das protocol. Actually, reacting $4(\mathrm{R}=\mathrm{Me})$ with $\mathrm{Ph}_{3} \mathrm{P} \cdot \mathrm{HBr}$, after careful filtration on silica gel eluting with ethyl acetate, we obtained a white phosphonium bromide salt in optimal yield (96\%). However, much to our surprise, its ${ }^{1} \mathrm{H}$ NMR was importantly different from the one reported by Das and his colleagues, which in our opinion was rather inconsistent. ${ }^{6}$ For example, the multiplicity and the integration of signals at $\delta 5.5-5.7$ are in accord for two protons; the integration of aromatics is excessive $\left({ }^{31} \mathrm{H}\right.$ instead of $\left.{ }^{15} \mathrm{H}\right)$, whereas that of methylenes appears deficient. Conversely, the above inconsistencies were not observed in the ${ }^{1} \mathrm{H}$ NMR recorded for the phosphonium salt in our hands to which we assigned the formula 9 that is the one of a "usual Wittig salt". 
From our point of view, no base-induced conversion of 11 to 10 was involved in the "anomalous Wittig reaction". More simply, our findings suggest that the ylide $\mathbf{1 0}$ originates from the salt 9, whose formation requires two classical chemical steps, namely, acid-catalyzed 1,4-dehydration of 4 , followed by the 1,4-addition of triphenylphosponium $\left(\mathrm{Ph}_{3} \mathrm{PH}^{+}\right)$onto the resulting diene $\mathbf{1 2}$ (Scheme 3).

Scheme 3. Proposed Mechanism (This Work) for the Conversion of 4 into Phosphonium Salt 9

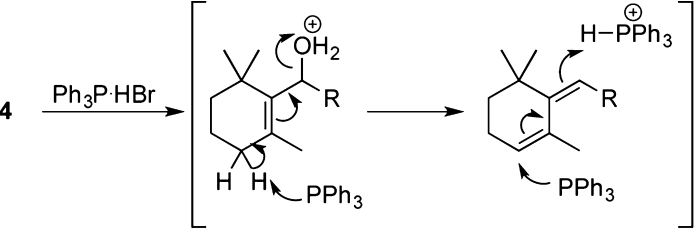

12

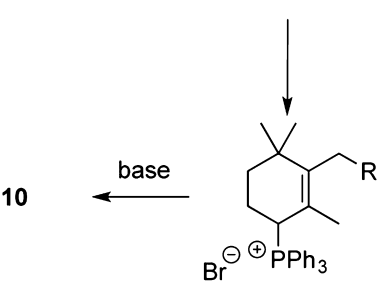

9

In this work, treatment of 4 with $\mathrm{PPh}_{3} \mathrm{HBr}$ gave cleanly, after careful filtration on silica gel (EtOAc elution, 96\% yield), a new white solid, which was unambiguously assigned as the phosphonium salt 9 (Scheme 3 ). The proton spectrum is depicted in Figure 2.

In order to definitively confirm the mechanism proposed above, we treated allylic alcohol 4 with hydrogen bromide in tert-butyl alcohol in the absence of triphenylphosphine, obtaining diene 12, which was isolated and fully characterized (Figure 3).

The subsequent exposure to triphenylphosphine hydrobromide in methanol afforded the phosphonium salt $\mathbf{9}$, thereby confirming our expectations (Scheme 4).

\section{CONCLUSIONS}

In conclusion, in this work, we provide convincing evidence that treatment of allylic alcohol 4 with $\mathrm{PPh}_{3} \cdot \mathrm{HBr}$ does not afford the phosphonium salt 11, as reported by Das and Evans, but the isomeric salt 9. Therefore, rationalization of the outcome of the Wittig reaction associated with such a phosphonium salt involves neither 1,2-P migrations nor 1,4$\mathrm{H}^{+}$shifts but simply an acid-promoted elimination and a regioselective addition to a protonated 1,3-diene. This latter mechanism is supported by strong analytical data such as monodimensional and bidimensional ${ }^{1} \mathrm{H}$ NMR spectra and appropriate corollary experiments.

\section{EXPERIMENTAL SECTION}

4.1. General Information. $\beta$-cyclocitral was purchased from commercial sources (Sigma-Aldrich, Milan, Italy). All other reagents and solvents were of the highest quality available or were freshly distilled. Melting points (uncorrected) were measured with a Buchi-Tottoli apparatus, and ${ }^{1} \mathrm{H},{ }^{13} \mathrm{C}$, ${ }^{31} \mathrm{P}$, and bidimensional NMR spectra were recorded on a

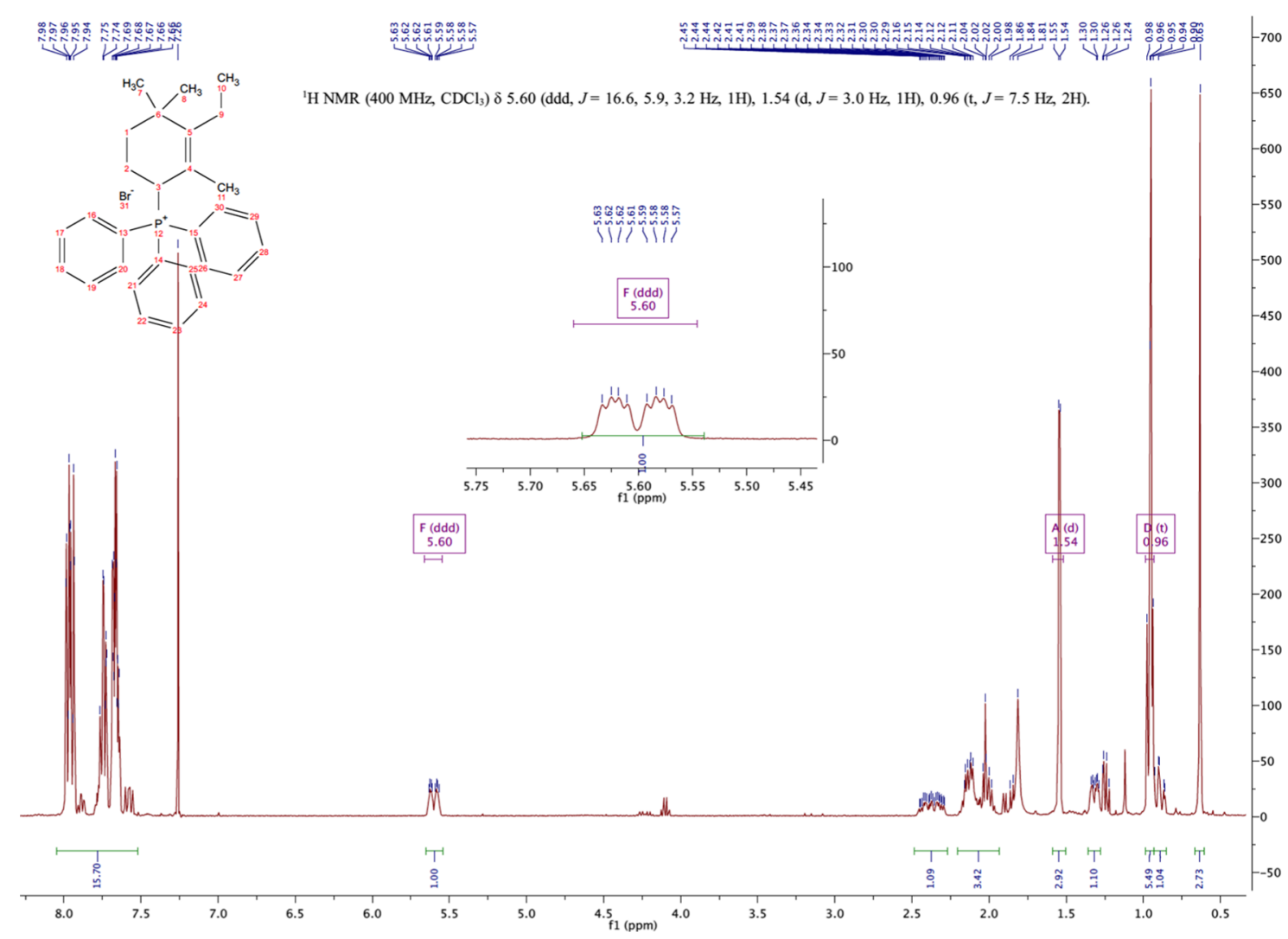

Figure 2. ${ }^{1} \mathrm{H}$ NMR spectra of phosphonium salt 9. 


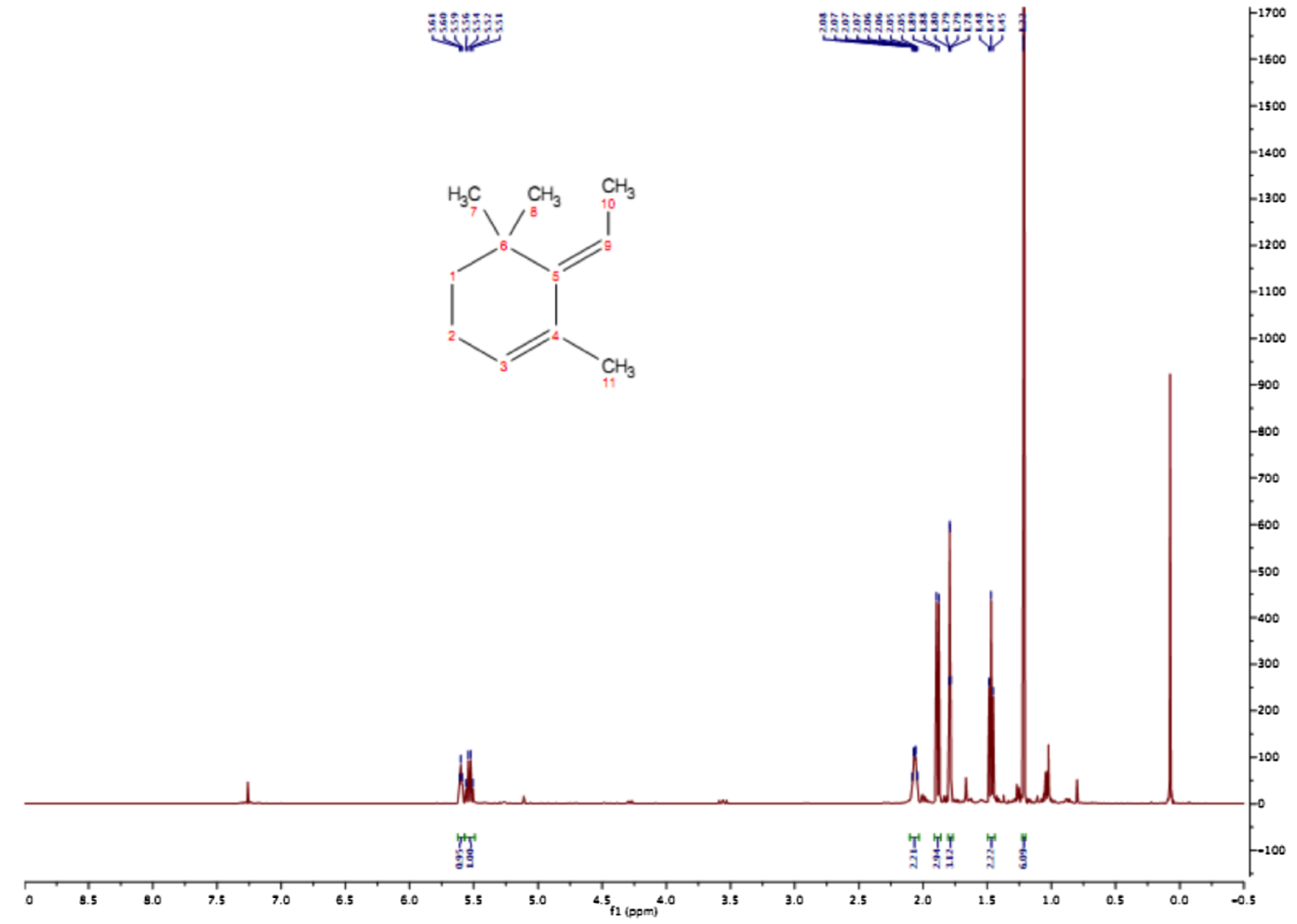

Figure 3. ${ }^{1} \mathrm{H}$ NMR spectra of diene $\mathbf{1 2}$.

Scheme 4. Conversion of 4 into 9 Passing through Isolated 1,3-Diene 12

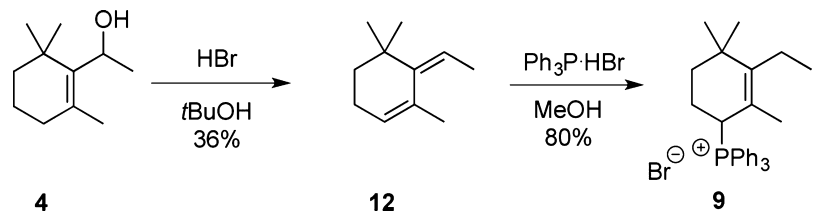

Varian $400 \mathrm{MHz}$ instrument unless otherwise noted. Chemical shifts are given in parts per million $(\delta)$ relative to the solvent, and coupling constants are in hertz. The abbreviations used are as follows: $\mathrm{s}=$ singlet, $\mathrm{d}=$ doublet, $\mathrm{t}=$ triplet, $\mathrm{q}=$ quartet, $\mathrm{dd}=$ double doublet, $\mathrm{m}=$ multiplet, $\mathrm{td}=$ triple doublet, $\mathrm{dt}=$ double triplet, and ddd = double double doublet. Mass spectrometry (MS) analyses were performed on ESI Micromass ZMD 2000. High-resolution MS (HRMS) spectra were recorded using an ESI-Q-TOF mass spectrometer (Agilent Technologies). Infrared spectra were recorded on a PerkinElmer FT-IR Spectrum 100 spectrometer. Flash chromatography was carried out on a silica gel (Merck, 230-400 mesh). High-performance liquid chromatography analysis was performed by Beckman system gold 168 using a C18 Jupiter column $(150 \times 4.6 \mathrm{~mm}, 5 \mu \mathrm{m})$ and water/acetonitrile/TFA eluent.

4.2. Experimental Procedures: Synthesis and Characterization. 4.2.1. (E)-4-((3-Ethyl-2,4,4-trimethylcyclohex-2en-1-ylidene)methyl)benzoic Acid (1). To a solution of compound $6(0.38 \mathrm{mmol}, 113 \mathrm{mg})$ dissolved in $4 \mathrm{~mL}$ of methanol was added a solution of $\mathrm{NaOH}(2 \mathrm{~N}, 1.14 \mathrm{mmol})$; the reaction was monitored by thin-layer chromatography (TLC) (eluent EtOAc/light petroleum 0.2/9.8) and ESI MS, and after $16 \mathrm{~h}$, hydrolysis was completed. The reaction was treated with $1 \mathrm{~N} \mathrm{HCl}$ until $\mathrm{pH} 1$ and extracted with $3 \times 15 \mathrm{~mL}$ of ethyl acetate. The organic layer was dried with sodium sulfate anhydrous and concentrated in vacuum. The crude acid (1) was crystallized from diethyl ether to afford the pure product in $74 \%$ yield.

(1): $\mathrm{C}_{19} \mathrm{H}_{24} \mathrm{O}_{2} ; \mathrm{mp} 150-155^{\circ} \mathrm{C}$. ESI $[\mathrm{MH}]^{+}$285.21. ESIQ-TOF exact mass: $[\mathrm{M}+\mathrm{H}]^{+}$calcd, 285.1849; found, 285.1839. ${ }^{1} \mathrm{H}$ NMR (400 MHz, MeOD): $\delta 7.84$ (d, $2 \mathrm{H}, J=7.6$ $\left.\mathrm{Hz}, \underline{\mathrm{CH}}_{\mathrm{ar}}\right), 7.20\left(\mathrm{~d}, 2 \mathrm{H}, J=7.6 \mathrm{~Hz}, \underline{\mathrm{C}}_{\mathrm{ar}}\right), 6.41(\mathrm{~s}, 1 \mathrm{H}, \mathrm{C} \underline{\mathrm{H}}=$ $\mathrm{C}_{\mathrm{q}}$ ), 2.54-2.52(m, $\left.2 \mathrm{H}, \mathrm{CH}_{2}-\mathrm{CH}_{2}-\mathrm{C}_{\mathrm{q}}=\right), 2.18(\mathrm{q}, 2 \mathrm{H}, \mathrm{J}=$ $\left.7.6 \mathrm{~Hz}, \mathrm{CH}_{2}-\mathrm{CH}_{3}\right), 1.85\left(\mathrm{~s}, 3 \mathrm{H}, \mathrm{CH}_{3}-\mathrm{C}=\right), 1.45-1.42(\mathrm{~m}$, $\left.2 \mathrm{H}, \mathrm{C}_{2}-\mathrm{CH}_{2}-\mathrm{C}_{\mathrm{q}}=\right), 1.03\left(\mathrm{~s}, 6 \mathrm{H}, \underline{\mathrm{CH}}_{3}-\mathrm{C}-\mathrm{C}_{3}\right), 1.02(\mathrm{t}$, $\left.3 \mathrm{H}, J=7.6 \mathrm{~Hz}, \mathrm{CH}_{2}-\mathrm{CH}_{3}\right) .{ }^{13} \mathrm{C} \mathrm{NMR}(100 \mathrm{MHz}, \mathrm{MeOD}): \delta$ $148.59\left(\mathrm{CH}_{3}-\mathrm{CH}_{2}-\underline{\mathrm{C}}=\right), 142.91\left(\mathrm{CO}-\underline{\mathrm{C}}_{\mathrm{qAr}}\right), 142.13$ $\left(\mathrm{CH}_{2}-\underline{\mathrm{C}}_{\mathrm{q}}=\mathrm{CH}\right), 134.92\left(\underline{\mathrm{C}}_{\mathrm{qAr}}-\mathrm{CH}=\mathrm{C}_{\mathrm{q}}\right), 130.12\left(2 \underline{\mathrm{C}} \mathrm{H}_{\mathrm{Ar}}\right)$, $129.75\left(2 \mathrm{CH}_{\mathrm{Ar}}\right), 128.41\left(=\underline{\mathrm{C}}_{\mathrm{q}}-\mathrm{CH}_{3}\right), 122.17\left(\mathrm{C}_{\mathrm{qAr}}-\underline{\mathrm{C}} \mathrm{H}=\right.$ $\left.\mathrm{C}_{\mathrm{q}}\right), 40.10\left(\mathrm{CH}_{2}-\mathrm{CH}_{2}-\mathrm{C}=\right), 36.80\left(\mathrm{CH}_{3}-\mathrm{C}_{\mathrm{q}}-\mathrm{CH}_{3}\right), 28.14$ $\left(2 \underline{\mathrm{CH}}_{3}\right), 25.31\left(\mathrm{CH}_{2}-\underline{\mathrm{CH}}_{2}-\mathrm{C}=\right), 23.73\left(\mathrm{CH}_{2}-\mathrm{CH}_{3}\right), 15.48$ $\left(\underline{\mathrm{CH}}_{3}-\mathrm{C}=\right.$ or $\left.\underline{\mathrm{CH}}_{3}-\mathrm{CH}_{2}\right), 15.21\left(\underline{\mathrm{CH}}_{3}-\mathrm{CH}_{2}\right.$ or $\left.\underline{\mathrm{CH}}_{3}-\mathrm{C}=\right)$. IR $\mathrm{cm}^{-1}$ : $2958.76 \mathrm{C}-\mathrm{H}$ stretching, $1669.14 \mathrm{C}=\mathrm{O}$ stretching, 1589.06 $\mathrm{C}=\mathrm{C}$ stretching, $961-610 \mathrm{C}-\mathrm{H}$ bending aromatic ring. X-ray: crystal data: $\mathrm{C}_{19} \mathrm{H}_{24} \mathrm{O}_{2}$; triclinic; space group: $P \overline{1}$; $Z=2 ; a=7.1637(2) \AA, b=7.6937(2) \AA, c=14.8938(4) \AA, \alpha$ $=82.749(1)^{\circ} ; \beta=81.158(1)^{\circ} ; \gamma=88.023(1)^{\circ} ; V=804.54(4)$ $\AA^{3}$; Mo K $\alpha$ radiation $\theta_{\text {max }}=27.5^{\circ}$; 3635 unique reflections measured; 2892 observed reflections $[I>2 \sigma(I)]$; final $R$ index $=0.0518$ (observed reflections), $R_{\mathrm{w}}=0.1518$ (all reflections).

4.2.2. (E)-N-Carbamimidoyl-4-((3-ethyl-2,4,4-trimethylcyclohex-2-en-1-ylidene)methyl) Benzamide (2). To a solution of guanidine hydrochloride $(2.66 \mathrm{mmol}, 254 \mathrm{mg})$ dissolved in a mixture of dimethylformamide (DMF)/dioxane (1:2), potassium tert-butoxide (2.66 mmol, $298 \mathrm{mg}$ ) was added 
portion wise. After $\mathrm{KCl}$ precipitation, the reaction was filtered and added to a solution of acid 1 ( $1.33 \mathrm{mmol}, 378 \mathrm{mg}$ ) dissolved in $10 \mathrm{~mL}$ of anhydrous DMF and carbonyl diimidazole (1.33 mmol, $216 \mathrm{mg}$ ) over argon atmosphere. After $15 \mathrm{~min}$, the solvent was removed under vacuum, and the crude solid was crystallized by water/methanol $1: 1$ at $0{ }^{\circ} \mathrm{C}$ to give a white solid in $77 \%$ yield.

(2): $\mathrm{C}_{20} \mathrm{H}_{27} \mathrm{~N}_{3} \mathrm{O} ; \mathrm{mp} 125-130{ }^{\circ} \mathrm{C}$. HRMS $[\mathrm{MH}]^{+}$ 326.22193. HRMS: $[\mathrm{MH}]^{+}$calcd, 326.22269. ${ }^{1} \mathrm{H}$ NMR (400 $\mathrm{MHz}$, acetone- $\left.d_{6}\right): \delta 8.04(\mathrm{br}, 3 \mathrm{H}, \mathrm{NH}), 7.98(\mathrm{~d}, 2 \mathrm{H}, J=8 \mathrm{~Hz}$, $\left.\mathrm{C}_{\mathrm{ar}}\right), 7.25\left(\mathrm{~d}, 2 \mathrm{H}, J=8 \mathrm{~Hz}, \mathrm{C}_{\mathrm{ar}}\right), 6.50\left(\mathrm{~s}, 1 \mathrm{H}, \mathrm{C} \underline{\mathrm{H}}=\mathrm{C}_{\mathrm{q}}\right)$,

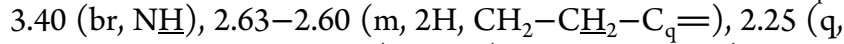
$\left.2 \mathrm{H}, J=7.6 \mathrm{~Hz}, \mathrm{CH}_{2}-\mathrm{CH}_{3}\right), 1.91\left(\mathrm{~s}, 3 \mathrm{H}, \mathrm{CH}_{3}-\mathrm{C}=\right)$, $1.49-$ $1.47\left(\mathrm{~m}, 2 \mathrm{H}, \mathrm{CH}_{2}-\mathrm{CH}_{2}-\mathrm{C}=\right), 1.08\left(\mathrm{~s}, 6 \mathrm{H}, 2 \underline{\mathrm{C}}_{3}\right), 1.04(\mathrm{t}$, $\left.3 \mathrm{H}, J=7.6 \mathrm{~Hz}, \mathrm{CH}_{2}-\mathrm{CH}_{3}\right) .{ }^{13} \mathrm{C}$ NMR $(100 \mathrm{MHz}$, acetone$\left.d_{6}\right): \delta 173.13(\mathrm{NH}-\underline{\mathrm{CO}}-\mathrm{Ar}), 160.62\left(\mathrm{NH}_{2}-\underline{\mathrm{C}}=\mathrm{NH}\right), 147.94$ $\left(\mathrm{CH}_{3} \mathrm{CH}_{2} \underline{\mathrm{C}}=\right), 141.41 \quad\left(2 \underline{\mathrm{C}}_{\mathrm{qAr}}\right), 141.14 \quad\left(\mathrm{CH}_{2}-\underline{\mathrm{C}}_{\mathrm{q}}=\mathrm{CH}\right)$, $130.05\left(2 \underline{\mathrm{CH}}_{\mathrm{Ar}}\right), 129.32\left(2 \underline{\mathrm{CH}}_{\mathrm{Ar}}\right), 128.10\left(\mathrm{CH}_{3}-\underline{\mathrm{C}}=\right)$, $122.30 \quad\left(\mathrm{C}_{\mathrm{qAr}}-\underline{\mathrm{CH}}=\mathrm{C}_{\mathrm{q}}\right), 39.68\left(\underline{\mathrm{CH}}_{2}-\mathrm{CH}_{2}-\mathrm{C}=\right), 36.46$ $\left(\mathrm{CH}_{3}-\underline{\mathrm{C}}_{\mathrm{q}}-\mathrm{CH}_{3}\right), 28.02\left(2 \underline{\mathrm{CH}}_{3}-\mathrm{C}_{\mathrm{q}}\right), 24.98\left(\mathrm{CH}_{2}-\underline{\mathrm{CH}}_{2}-\right.$ $\mathrm{C}=), 23.39\left(\underline{\mathrm{CH}}_{2}-\mathrm{CH}_{3}\right), 15.48\left(\underline{\mathrm{CH}}_{3}-\mathrm{C}=\right), 15.17\left(\mathrm{CH}_{2}-\right.$ $\left.\mathrm{CH}_{3}\right)$.

4.2.3. 1-(2,6,6-Trimethylcyclohex-1-yl) Ethanol (4). To a solution of $\beta$-cyclocitral $3(6.56 \mathrm{mmol}, 1 \mathrm{~g})$ in $20 \mathrm{~mL}$ of anhydrous tetrahydrofuran (THF), methylmagnesium bromide $(7.22 \mathrm{mmol}, 5.16 \mathrm{~mL})$ was added at $0{ }^{\circ} \mathrm{C}$. The reaction was monitored by TLC (eluent: EtOAc/light petroleum 1/6) and was completed after $12 \mathrm{~h}$. The reaction was quenched with a saturated solution of ammonium chloride, THF was evaporated in vacuo, and the crude material was dissolved in ethyl acetate. The organic phases were washed with water, separated, and dried over sodium sulfate anhydrous. The crude alcohol was purified by flash chromatography using AcOEt/ light petroleum $1 / 9$ as the eluent, furnishing a pale yellow oil with $78 \%$ yield.

(4): $\mathrm{C}_{11} \mathrm{H}_{20} \mathrm{O}$. ESI $[\mathrm{MH}]^{+}$169.28. IR cm $\mathrm{cm}^{-1}: 3378.96 \mathrm{OH}$ stretching, $2906.78 \mathrm{C}-\mathrm{H}$ stretching, $1652.40 \mathrm{C}=\mathrm{C}$ stretching, $1064.62 \mathrm{C}-\mathrm{O}$ stretching. ${ }^{1} \mathrm{H} \mathrm{NMR}\left(400 \mathrm{MHz}, \mathrm{CDCl}_{3}\right): \delta 4.50$ (q) $\left.1 \mathrm{H}, J=6.7 \mathrm{~Hz}, \mathrm{CH}_{3}-\mathrm{CH}-\mathrm{OH}\right), 1.92$ (dd, $2 \mathrm{H}, J=13.9$, $\left.6.5 \mathrm{~Hz}, \mathrm{CH}_{2}-\mathrm{CH}_{2}-\mathrm{CH}_{2}\right), 1.85\left(\mathrm{~s}, 3 \mathrm{H}, \mathrm{CH}_{3}-\mathrm{C}=\mathrm{C}\right), 1.57-$ $1.37\left(\mathrm{~m}, 2 \mathrm{H}, \mathrm{CH}_{2}-\mathrm{CH}_{2}-\mathrm{CH}_{2}\right), 1.41(\mathrm{~d}, 3 \mathrm{H}, J=6.7 \mathrm{~Hz}$, $\mathrm{CH}_{3}-\mathrm{CH}-\mathrm{OH}$ ), $1.40-1.37$ (m, $\left.2 \mathrm{H}, \mathrm{CH}_{2}-\mathrm{CH}_{2}-\mathrm{CH}_{2}\right), 1.09$ (s, $\left.3 \mathrm{H}, \mathrm{CH}_{3}-\mathrm{C}_{\mathrm{q}}-\mathrm{CH}_{3}\right), 0.95\left(\mathrm{~s}, 3 \mathrm{H}, \mathrm{CH}_{3}-\mathrm{C}_{\mathrm{q}}-\mathrm{CH}_{3}\right) .{ }^{13} \mathrm{C}$ NMR $\left(100 \mathrm{MHz}, \mathrm{CDCl}_{3}\right): \delta 141.31\left(\mathrm{CH}-\underline{\mathrm{C}}_{\mathrm{q}}=\mathrm{C}_{\mathrm{q}}-\mathrm{CH}_{3}\right)$, $130.97\left(\mathrm{CH}-\mathrm{C}_{\mathrm{q}}=\underline{\mathrm{C}}_{\mathrm{q}}-\mathrm{CH}_{3}\right), 67.18\left(\mathrm{CH}_{3}-\underline{\mathrm{CH}}-\mathrm{OH}\right), 40.02$ $\left(\mathrm{C}_{\mathrm{q}}-\mathrm{CH}_{2}-\mathrm{CH}_{2}-\mathrm{CH}_{2}\right), 34.70\left(2 \mathrm{CH}_{3}-\underline{\mathrm{C}}_{\mathrm{q}}\right), 34.13\left(\mathrm{C}_{\mathrm{q}}-\mathrm{CH}_{2}-\right.$ $\left.\mathrm{CH}_{2}-\underline{\mathrm{CH}}_{2}\right), 28.64\left(\underline{\mathrm{CH}}_{3}-\mathrm{C}_{\mathrm{q}}-\mathrm{CH}_{3}\right), 28.01\left(\mathrm{CH}_{3}-\mathrm{C}_{\mathrm{q}}-\underline{\mathrm{CH}}_{3}\right)$, $23.13\left(\underline{\mathrm{CH}}_{3}-\mathrm{CH}-\mathrm{OH}\right), 21.03\left(\underline{\mathrm{CH}}_{3}-\mathrm{C}_{\mathrm{q}}=\mathrm{C}_{\mathrm{q}}\right), 19.37\left(\mathrm{C}_{\mathrm{q}}-\right.$ $\left.\mathrm{CH}_{2}-\mathrm{CH}_{2}-\mathrm{CH}_{2}\right)$.

4.2.4. (E)-Methyl 4-((3-Ethyl-2,4,4-trimethylcyclohex-2-en1-ylidene)methyl)benzoate (6). To a solution of compound 9 $(2.13 \mathrm{mmol}, 1.05 \mathrm{~g})$ in anhydrous THF, $0.85 \mathrm{~mL}$ of $n$-BuLi $(2.5 \mathrm{M}, 2.13 \mathrm{mmol})$ was added; after $30 \mathrm{~min}$, the temperature was cooled at $-78{ }^{\circ} \mathrm{C}$, and methyl-formyl benzoate $(2.34$ mmol, $384 \mathrm{mg}$ ) was added. The reaction was mixed overnight, followed by TLC (eluent EtOAc/light petroleum 0.2/9.8); the reaction was quenched with a saturated solution of ammonium chloride, concentrated in vacuo, dissolved in $30 \mathrm{~mL}$ of ethyl acetate, and washed with water $(2 \times 30 \mathrm{~mL})$. The organic layer was dried, concentrated in vacuum, and purified by flash chromatography (eluent EtOAc/light petroleum 0.2/9.8) to afford compound 6 as a dark/brown oil in $26 \%$ yield.
(6): $\mathrm{C}_{20} \mathrm{H}_{26} \mathrm{O}_{2}$. ESI $[\mathrm{MH}]^{+}$299.41. IR cm $\mathrm{cm}^{-1} 2952 \mathrm{C}-\mathrm{H}$ stretching, $1717 \mathrm{C}=\mathrm{O}$ stretching, $1602 \mathrm{C}=\mathrm{C}$ stretching, 1102 $\mathrm{C}-\mathrm{O}-\mathrm{C}$ stretching; 961-610 $\mathrm{C}-\mathrm{H}$ aromatic bending. ${ }^{1} \mathrm{H}$ $\operatorname{NMR}\left(400 \mathrm{MHz}, \mathrm{CDCl}_{3}\right): \delta 7.95\left(\mathrm{~d}, 2 \mathrm{H}, J=8.4 \mathrm{~Hz}, \mathrm{H}_{\text {ar. }}\right)$, $7.32\left(\mathrm{~d}, 2 \mathrm{H}, J=8.2 \mathrm{~Hz}, \mathrm{H}_{\mathrm{ar} .}\right), 6.40\left(\mathrm{~s}, 1 \mathrm{H}, \mathrm{C}_{\mathrm{q}}-\mathrm{CH}=\mathrm{C}_{\mathrm{q}}\right), 3.95$ $\left(\mathrm{s}, 3 \mathrm{H}, \mathrm{OCH}_{3}\right), 2.6\left(\mathrm{ddd}, 2 \mathrm{H}, J=8.2,4.7,1.7 \mathrm{~Hz}, \mathrm{CH}_{2}-\mathrm{CH}_{2}-\right.$ $\left.\mathrm{C}_{\mathrm{q}}=\right), 2.20\left(\mathrm{q}, 2 \mathrm{H}, \mathrm{J}=7.2 \mathrm{~Hz}, \mathrm{CH}_{2}-\mathrm{CH}_{3}\right), 1.95\left(\mathrm{~s}, 3 \mathrm{H}, \mathrm{C}_{\mathrm{q}}-\right.$ $\left.\mathrm{CH}_{3}\right), 1.50\left(\mathrm{~m}, 2 \mathrm{H}, \mathrm{CH}_{2}-\mathrm{CH}_{2}-\mathrm{C}_{\mathrm{q}}=\right), 1.15(\mathrm{t}, 3 \mathrm{H}, J=7.2$ $\left.\mathrm{Hz}, \mathrm{CH}_{2}-\mathrm{CH}_{3}\right), 1.15\left(\mathrm{~s}, 6 \mathrm{H}, \mathrm{CH}_{3}-\mathrm{C}_{\mathrm{q}}-\mathrm{CH}_{3}\right) .{ }^{13} \mathrm{C}$ NMR (100 $\left.\mathrm{MHz}, \mathrm{CDCl}_{3}\right): \delta 167.20(\underline{\mathrm{C}}=\mathrm{O}), 148.91\left(\mathrm{CH}_{3}-\mathrm{CH}_{2}-\underline{\mathrm{C}}=\right)$, $144.00\left(\underline{\mathrm{C}}_{\mathrm{qAr}}\right), 142.29\left(\mathrm{CH}_{2}-\underline{\mathrm{C}}_{\mathrm{q}}=\mathrm{CH}\right), 136.07\left(\underline{\mathrm{CH}}_{\mathrm{Ar}}\right)$, $129.95\left(\underline{\mathrm{CH}}_{\mathrm{Ar}}\right), 129.30\left(\underline{\mathrm{CH}}_{\mathrm{Ar}}\right), 127.12\left(=\underline{\mathrm{C}}_{\mathrm{q}}-\mathrm{CH}_{3}\right), 126.17$ $\left(\underline{\mathrm{CH}}_{\mathrm{Ar}}\right), 120.57 \quad\left(\mathrm{C}_{\mathrm{qAr}}-\underline{\mathrm{C}} \mathrm{H}=\mathrm{C}_{\mathrm{q}}\right), 52.06\left(\mathrm{O}-\underline{\mathrm{C}} \mathrm{H}_{3}\right), 38.84$ $\left(\mathrm{CH}_{2}-\mathrm{CH}_{2}-\mathrm{C}=\right), 35.95\left(\mathrm{CH}_{3}-\underline{\mathrm{C}}_{\mathrm{q}}-\mathrm{CH}_{3}\right), 27.69\left(2 \mathrm{CH}_{3}\right)$, $24.36\left(\mathrm{CH}_{2}-\mathrm{CH}_{2}-\mathrm{C}=\right), 22.99\left(\mathrm{CH}_{2}-\mathrm{CH}_{3}\right), 15.28\left(\mathrm{CH}_{3}-\right.$ $\mathrm{C}=$ or $\left.\underline{\mathrm{CH}}_{3}-\mathrm{CH}_{2}\right), 14.80\left(\underline{\mathrm{CH}}_{3}-\mathrm{CH}_{2}\right.$ or $\left.\underline{\mathrm{CH}}_{3}-\mathrm{C}=\right)$.

4.2.5. 4-Ethyl-3,5,5-trimethyl-2-(triphenylphosphonio)cyclohex-3-en-1-ide (9). 4.2.5.1. Starting from Compound 4. A solution of allylic alcohol $4(2.97 \mathrm{mmol}, 500 \mathrm{mg})$ in 30 $\mathrm{mL}$ of degassed methanol was treated with triphenylphosphine hydrobromide $(2.97 \mathrm{mmol}, 1018 \mathrm{mg}$ ), and the reaction was mixed overnight. The reaction was monitored by ESI MS (that showed the presence of phosphonium salt $[\mathrm{M}]^{+}=413.24$, triphenylphosphine, and triphenylphosphine oxide) and TLC (eluent EtOAc/light petroleum 0.2/9.8); when the reaction was completed, methanol was evaporated under reduced pressure; and the crude yellow solid was purified by silica gel filtration using ethyl acetate as the eluent to obtain a white solid in $96 \%$ yield.

4.2.5.2. Starting from Compound 12. A solution of diene $12(0.5 \mathrm{mmol}, 75 \mathrm{mg})$ in $5 \mathrm{~mL}$ of degassed methanol was treated with triphenylphosphine hydrobromide $(0.5 \mathrm{mmol}, 170$ $\mathrm{mg}$ ), and the reaction was mixed overnight. The reaction was monitored by ESI MS; at the end, methanol was evaporated under reduced pressure; and the crude yellow solid was purified by silica gel filtration using ethyl acetate as the eluent to obtain the phosphonium salt as a white solid in $80 \%$ yield.

(9): $\mathrm{C}_{29} \mathrm{H}_{34} \mathrm{BrP} ; \mathrm{mp} 220{ }^{\circ} \mathrm{C}$ with decomposition. ESI $[\mathrm{MH}]^{+}$413. HRMS: $\left[\mathrm{M}^{+}\right]$calcd, 413.2398. HRMS: $\left[\mathrm{M}^{+}\right]$ 413.2406. IR $\mathrm{cm}^{-1}: 3387.91 \mathrm{C}-\mathrm{H}$ aromatic stretching, $2961.96 \mathrm{C}-\mathrm{H}$ olefin stretching, $1586.62 \mathrm{C}=\mathrm{C}$ stretching, 1437.04 $\mathrm{C}-\mathrm{H}$ olefin bending, 691.22-921.59 $\mathrm{C}-\mathrm{H}$ bending. ${ }^{1} \mathrm{H}$ NMR (400 MHz, $\mathrm{CDCl}_{3}$ ): $\delta 8.01-7.55\left(\mathrm{~m}, 15 \mathrm{H}, \underline{\mathrm{Ph}}_{3}-\mathrm{P}\right)$, 5.60 (ddd, $\left.1 \mathrm{H}, J=16.6,5,3.2 \mathrm{~Hz}, \mathrm{C} \underline{\mathrm{H}}-\mathrm{P}^{+}\left(\mathrm{Ph}_{3}\right)\right)$, 2.49-2.40 (m, 1H, $\left.\underline{\mathrm{CH}}_{2}-\mathrm{CH}-\mathrm{P}\right), 2.20-2.10\left(\mathrm{~m}, 3 \mathrm{H}, \mathrm{CH}_{2}-\mathrm{CH}-\mathrm{P}, \mathrm{C}_{\mathrm{q}}-\right.$ $\left.\mathrm{CH}_{2}-\mathrm{CH}_{2}-\mathrm{CH}\right), 1.54$ (d, $3 \mathrm{H}, J=3 \mathrm{~Hz}, \mathrm{CH}_{3}-\mathrm{C}_{\mathrm{q}}=\mathrm{C}_{\mathrm{q}}$ ), 1.32 (ddd, $\left.1 \mathrm{H}, J=13.3,5.5,2.8 \mathrm{~Hz}, \mathrm{CH}_{2}-\mathrm{CH}_{3}\right), 0.96(\mathrm{~s}, 3 \mathrm{H}$, $\left.\mathrm{CH}_{3}-\mathrm{C}_{\mathrm{q}}-\mathrm{CH}_{3}\right), 0.95\left(\mathrm{t}, 3 \mathrm{H}, J=7.5 \mathrm{~Hz}, \mathrm{CH}_{2}-\mathrm{CH}_{3}\right), 0.94(\mathrm{~m}$, $\left.1 \mathrm{H}, \mathrm{CH}_{2}-\mathrm{CH}_{3}\right), 0.63\left(\mathrm{~s}, 3 \mathrm{H}, \mathrm{CH}_{3}-\mathrm{C}_{\mathrm{q}}-\mathrm{CH}_{3}\right) .{ }^{13} \mathrm{C} \mathrm{NMR}(100$ $\left.\mathrm{MHz}, \mathrm{CDCl}_{3}\right): \delta 149.85\left(\mathrm{C}_{\mathrm{q}}=\mathrm{C}_{\mathrm{q}}-\mathrm{CH}_{2}-\mathrm{CH}_{3}\right), 149.74$ $\left(\underline{\mathrm{C}}_{\mathrm{qAr}}\right), 134.57\left(\underline{\mathrm{CH}}_{\mathrm{Ar}}\right), 130.80\left(\underline{\mathrm{CH}}_{\mathrm{Ar}}\right), 130.17\left(\mathrm{CH}_{\mathrm{Ar}}\right)$, $119.97\left(\underline{\mathrm{C}}_{\mathrm{qAr}}\right), 119.18\left(\mathrm{C}_{\mathrm{q}}=\underline{\mathrm{C}}_{\mathrm{q}}-\mathrm{CH}_{3}\right), 40.17(\underline{\mathrm{CH}}-$ $\left.\mathrm{P}^{+}\left(\mathrm{Ph}_{3}\right)\right), 35.56\left(\mathrm{C}_{\mathrm{q}}-\underline{\mathrm{CH}_{2}}-\mathrm{CH}_{3}\right), 35.42\left(\mathrm{CH}_{3}-\underline{\mathrm{C}}_{\mathrm{q}}-\mathrm{CH}_{3}\right)$, $27.82\left(\underline{\mathrm{CH}}_{3}-\mathrm{C}_{\mathrm{q}}-\mathrm{CH}_{3}\right), 27.51\left(\mathrm{CH}_{3}-\mathrm{C}_{\mathrm{q}}-\underline{\mathrm{CH}}_{3}\right), 22.64\left(\mathrm{C}_{\mathrm{q}}-\right.$ $\mathrm{CH}_{2}-\mathrm{CH}_{2}-\mathrm{CH}$ or $\left.\mathrm{C}_{\mathrm{q}}-\mathrm{CH}_{2}-\mathrm{CH}_{2}-\mathrm{CH}\right), 21.81\left(\mathrm{C}_{\mathrm{q}}-\mathrm{CH}_{2}-\right.$ $\underline{\mathrm{CH}_{2}}-\mathrm{CH}$ or $\left.\mathrm{C}_{\mathrm{q}}-\underline{\mathrm{CH}}_{2}-\mathrm{CH}_{2}-\mathrm{CH}\right), 21.53\left(\mathrm{C}_{\mathrm{q}}=\mathrm{C}_{\mathrm{q}}-\mathrm{CH}_{3}\right)$, $13.78\left(\mathrm{C}_{\mathrm{q}}-\mathrm{CH}_{2}-\mathrm{CH}_{3}\right)$.

4.2.6. (E)-6-Ethylidene-1,5,5-trimethylcyclohex-1-ene (12). To a $0.1 \mathrm{M}$ solution of compound $4(0.59 \mathrm{mmol}, 100 \mathrm{mg})$, in tert-butanol $(6 \mathrm{~mL}) 0.1 \mathrm{~mL}$ of $\mathrm{HBr}$ solution $(5.7 \mathrm{M}$ in acetic acid, $0.59 \mathrm{mmol}$ ) was added. After $30 \mathrm{~min}$, the reaction was completed (TLC eluent EtOAc/light petroleum 0.2/9.8), a 2 $\mathrm{N}$ solution of $\mathrm{NaOH}$ was added until basic $\mathrm{pH}$, the solvent was 
evaporated under vacuum, and the crude material was dissolved in $5 \mathrm{~mL}$ of water. The aqueous phase was extracted twice with ethyl acetate $(20 \mathrm{~mL}$ each $)$, dried under sodium sulfate, and concentrated in vacuum. Diene 12 was obtained in $36 \%$ yield after flash chromatography purification using EtOAc/light petroleum $0.2 / 9.8$ as the eluent.

(12): $\mathrm{C}_{11} \mathrm{H}_{18}$. ESI $[\mathrm{MH}]^{+}$151.29. ${ }^{1} \mathrm{H}$ NMR (400 MHz, $\left.\mathrm{CDCl}_{3}\right): \delta 5.60\left(\mathrm{~m}, 1 \mathrm{H}, \mathrm{CH}=\mathrm{C}_{\mathrm{q}}-\mathrm{CH}_{3}\right), 5.53(\mathrm{q}, 1 \mathrm{H}, J=7.5$ $\left.\mathrm{Hz}, \mathrm{C} \underline{\mathrm{H}}-\mathrm{CH}_{3}\right), 2.15\left(\mathrm{~m}, 2 \mathrm{H}, \mathrm{CH}_{2}-\mathrm{CH}_{2}-\mathrm{CH}\right), 1.95$ (d, 3H, J $\left.=7.2 \mathrm{~Hz}, \mathrm{CH}-\mathrm{CH}_{3}\right), 1.79\left(\mathrm{~s}, 3 \mathrm{H}, \mathrm{CH}_{3}-\mathrm{C}=\right), 2.45(\mathrm{t}, 2 \mathrm{H}, J=$ $12.2 \mathrm{~Hz}, \mathrm{CH}_{2}-\mathrm{CH}_{2}-\mathrm{CH}$ ), 1.20 (s, $\left.6 \mathrm{H}, \mathrm{C}_{3}-\mathrm{C}_{\mathrm{q}}-\mathrm{C}_{3}\right) .{ }^{13} \mathrm{C}$ NMR $\left(100 \mathrm{MHz}, \mathrm{CDCl}_{3}\right): \delta 144.34\left(\mathrm{C}_{\mathrm{q}}=\mathrm{CH}-\mathrm{CH}_{3}\right), 133.67$ $\left(\mathrm{CH}_{3}-\mathrm{C}_{\mathrm{q}}=\mathrm{CH}\right), 125.20\left(\mathrm{C}_{\mathrm{q}}=\underline{\mathrm{CH}}-\mathrm{CH}_{3}\right), 119.47\left(\mathrm{CH}_{3}-\right.$ $\left.\mathrm{C}_{\mathrm{q}}=\underline{\mathrm{CH}}\right), 40.48\left(\mathrm{C}_{\mathrm{q}}-\underline{\mathrm{CH}}_{2}-\mathrm{CH}_{2}\right), 34.79\left(\mathrm{CH}_{3}-\underline{\mathrm{C}}_{\mathrm{q}}-\mathrm{CH}_{3}\right)$, $27.88\left(2 \mathrm{CH}_{3}\right), 22.95\left(\mathrm{CH}_{2}-\mathrm{CH}_{2}-\mathrm{CH}\right), 21.88\left(\mathrm{C}_{\mathrm{q}}=\mathrm{CH}-\right.$ $\left.\mathrm{CH}_{3}\right), 16.14\left(\mathrm{C}_{\mathrm{q}}=\mathrm{CH}-\underline{\mathrm{CH}}_{3}\right)$.

\section{ASSOCIATED CONTENT}

\section{S Supporting Information}

The Supporting Information is available free of charge on the ACS Publications website at DOI: 10.1021/acsomega. 8 b01228.

Representative experimental procedure and monodimensional and bidimensional NMR and HRMS spectra (PDF)

\section{AUTHOR INFORMATION}

\section{Corresponding Author}

*E-mail: trap@unife.it. Phone: 0039-0532-455924. Fax: 00390532-455953.

\section{ORCID}

Claudio Trapella: 0000-0002-6666-143X

\section{Author Contributions}

${ }^{\dagger}$ A.F. and P.M. contributed equally.

\section{Notes}

The authors declare no competing financial interest.

\section{ACKNOWLEDGMENTS}

We are grateful to Dr. Alberto Casolari and Dr. ssa Elisa Durini for the NMR analysis, Dr. Erika Marzola for the MS analysis, and Prof. Gian Piero Pollini and Prof. Giovanni Poli for the successful discussion about the phosphonium mechanism formation.

\section{REFERENCES}

(1) Das, B. C.; Kabalka, G. W. Design and synthesis of (E)-4-((3ethyl-2,4,4-trimethylcyclohex-2-enylidene)methyl)benzoic acid. Tetrahedron Lett. 2008, 49, 4695.

(2) Das, B. C.; Smith, M. E.; Kalpana, G. V. Design, synthesis of novel peptidomimetic derivatives of 4-HPR for rhabdoid tumors. Bioorg. Med. Chem. Lett. 2008, 18, 4177-4180.

(3) Das, B. C.; Tang, X.-Y.; Sanyal, S.; Mohapatra, S.; Rogler, P.; Nayak, S.; Evans, T. Design and synthesis of 3,5-disubstituted 1,2,4oxadiazole containing retinoids from a retinoic acid receptor agonist. Tetrahedron Lett. 2011, 52, 2433.

(4) (a) Jubinsky, P. T.; Short, M. K.; Ghanem, M.; Das, B. C. Design, synthesis, and biological activity of novel Magmas inhibitors. Bioorg. Med. Chem. Lett. 2011, 21, 3479. (b) Zatelli, M. C.; Gagliano, T.; Pelà, M.; Bianco, S.; Bertolasi, V.; Tagliati, F.; Guerrini, R.; degli Uberti, E.; Salvadori, S.; Trapella, C. N-Carbamidoyl-4-((3-ethyl-2,4,4 -trimethylcyclohexyl)methyl)benzamide Enhances Staurosporine Cytotoxic Effects Likely Inhibiting the Protective Action of Magmas toward Cell Apoptosis. J. Med. Chem. 2014, 57, 4606-4614.
(5) Rosenberger, M.; Neukom, C. Retinoic Acid Metabolites. 3 Total Synthesis of (2E,4E,6E,8E)-3,7-Dimethyl-9-[6,6-dimethyl-2(hydroxymethyI)-1-cyclohexen-1-yI]-2,4,6,8-nonatetraenoic Acid. J. Org. Chem. 1982, 47, 1782-1785. Das, B. C.; Mahalingam, S. M.; Evans, T.; Kabalka, G. W.; Anguiano, J.; Hema, K. Design and synthesis of (E)-1-((3-ethyl-2,4,4-trimethylcyclohex-2-enylidene)methyl-4-substituted benzenes from 1-(2,6,6-trimethylcyclohex-1enyl)ethanol. Chem. Commun. 2009, 2133-2135.

(6) For a precedent of acid-promoted conversion of an allylic alcohol into a 1,3-diene see: De Marco, R.; Leggio, A.; Liguori, A.; Perri, F.; Siciliano, C. Transformations of 3-Hydroxy Steroids with Lewis and Anhydrous Protic Acids: The Case of Pregn-4-en-3b,17a,20b-Triol. Chem. Biol. Drug Des. 2011, 78, 269.

(7) Das, B. C.; Mahalingam, S. M.; Panda, L.; Wang, B.; Campbell, P. D.; Evans, T. Design and synthesis of potential new apoptosis agents: hybrid compounds containing perillyl alcohol and new constrained retinoids. Tetrahedron Lett. 2010, 51, 1462-1466. 